\title{
Experience in the treatment of pulmonary nocardiosis: A report of a fatal case
}

\author{
YUHONG LI ${ }^{1}$, HONG LIU ${ }^{1}$ and YOULU SHEN ${ }^{2}$ \\ Departments of ${ }^{1}$ Respiratory Medicine, and ${ }^{2}$ Cardiovascular Medicine, \\ Qinghai University Affiliated Hospital, Xining, Qinghai 810001, P.R. China
}

Received February 27, 2021; Accepted April 23, 2021

DOI: 10.3892/wasj.2021.104

\begin{abstract}
Pulmonary nocardiosis is an opportunistic infection that is commonly observed in immunocompromised individuals and in those with chronic disease. The present study reports the case of a female patient who was diagnosed with an intermuscular abscess in the left leg and with pulmonary nocardiosis. The patient had a 20-year history of the use of paracetamol and codeine phosphate drugs for the alleviation of headaches. The patient was treated with linezolid and trimethoprim-sulfamethoxazole (TMP-SMX). The symptoms of the patient improved and the patient was discharged 14 days later. The drug was discontinued spontaneously following discharge and the patient then passed away two months later. On the whole, the present study highlights the importance of the early diagnosis and standard treatment for pulmonary nocardiosis.
\end{abstract}

\section{Introduction}

Nocardia is a genus of bacteria that can cause a wide variety of opportunistic infections, ranging from localized cutaneous and pulmonary infections to deep-seated and disseminated infections in immunosuppressed patients $(1,2)$. However, to date, at least to the best of our knowledge, there is no published report of infections caused by paracetamol and codeine phosphate. The present study describes the case of a female patient diagnosed and treated for pulmonary nocardiosis, who eventually succumbed to the disease. The experience encountered with the patient in question and the knowledge acquired from the case are also discussed.

Correspondence to: Dr Youlu Shen, Department of Cardiovascular Medicine, Qinghai University Affiliated Hospital, 29 Tongren Road, Xining, Qinghai 810001, P.R. China

E-mail: 452383473@qq.com

Key words: nocardiosis, paracetamol and codeine phosphate, experience

\section{Case report}

A 77-year-old woman was admitted to Haidong Municipal Hospital (Pingan, Qinghai) with complaints of leg swelling and pain for one month, and a fever and cough for 10 days. Ceftazidime and metronidazole injection were administered and the symptoms improved after one week. Treatment was thus suspended for 10 days. However, the patient developed a fever, and suffered from chills and a cough, with a body temperature of $>39^{\circ} \mathrm{C}$. The sputum of the patient was yellow and purulent. She had no history of smoking, hypertension, diabetes or any other immune-related diseases. However, the patient had been using paracetamol and codeine phosphate at a daily dose of 4-8 pills (each tablet contains $500 \mathrm{mg}$ of acetaminophen and $8.4 \mathrm{mg}$ of codeine phosphate) over the past 20 years for the alleviation of headaches.

A physical examination revealed a pulse rate of $102 / \mathrm{min}$ and a body temperature of $38.2^{\circ} \mathrm{C}$. Diffuse rales were heard throughout both lungs. Evident swelling was observed in the left leg with an increase in temperature. A laboratory examination revealed a white blood cell count of $11.46 \times 10^{9} / 1$, a neutrophil ratio of $84 \%$, an erythrocyte sedimentation rate of $90 \mathrm{~mm} / \mathrm{h}$, a C-reactive protein concentration of $120 \mathrm{mg} / \mathrm{l}$ and a procalcitonin concentration of $7.8 \mathrm{ng} / \mathrm{ml}$. Nocardia was identified in the culture of the rinse solution from the left leg. A chest computed tomography (CT) scan revealed multiple irregular thick wall cavities along with the mass, patch, cords and nodular shadows at the beginning (Fig. 1A). The magnetic resonance imaging (MRI) of the left leg revealed an intermuscular abscess (Fig. 2).

The patient was treated with linezolid ( $0.6 \mathrm{~g}$, every $12 \mathrm{~h}$ via intravenous drip) for 10 days at Qinghai University Affiliated Hospital (the patient had then been transferred to this hospital), and the symptoms significantly improved. The multiple irregular thick wall cavities were significantly absorbed, as revealed by a chest CT scan (Fig. 1B). After three days, the patient developed symptoms of right gum pain and swelling in the left leg again. Drainage was performed in the aching gum and intermuscular abscess, and Nocardia was identified in the culture of the rinse solution. Oral trimethoprim-sulfamethoxazole (TMP-SMX) was administered. The symptoms then disappeared and the patient was discharged 14 days later. The lesions had significantly shrunk in size, as shown by the chest CT scan (Fig. 1C); however, magnetic resonance spectroscopy of the left leg was 

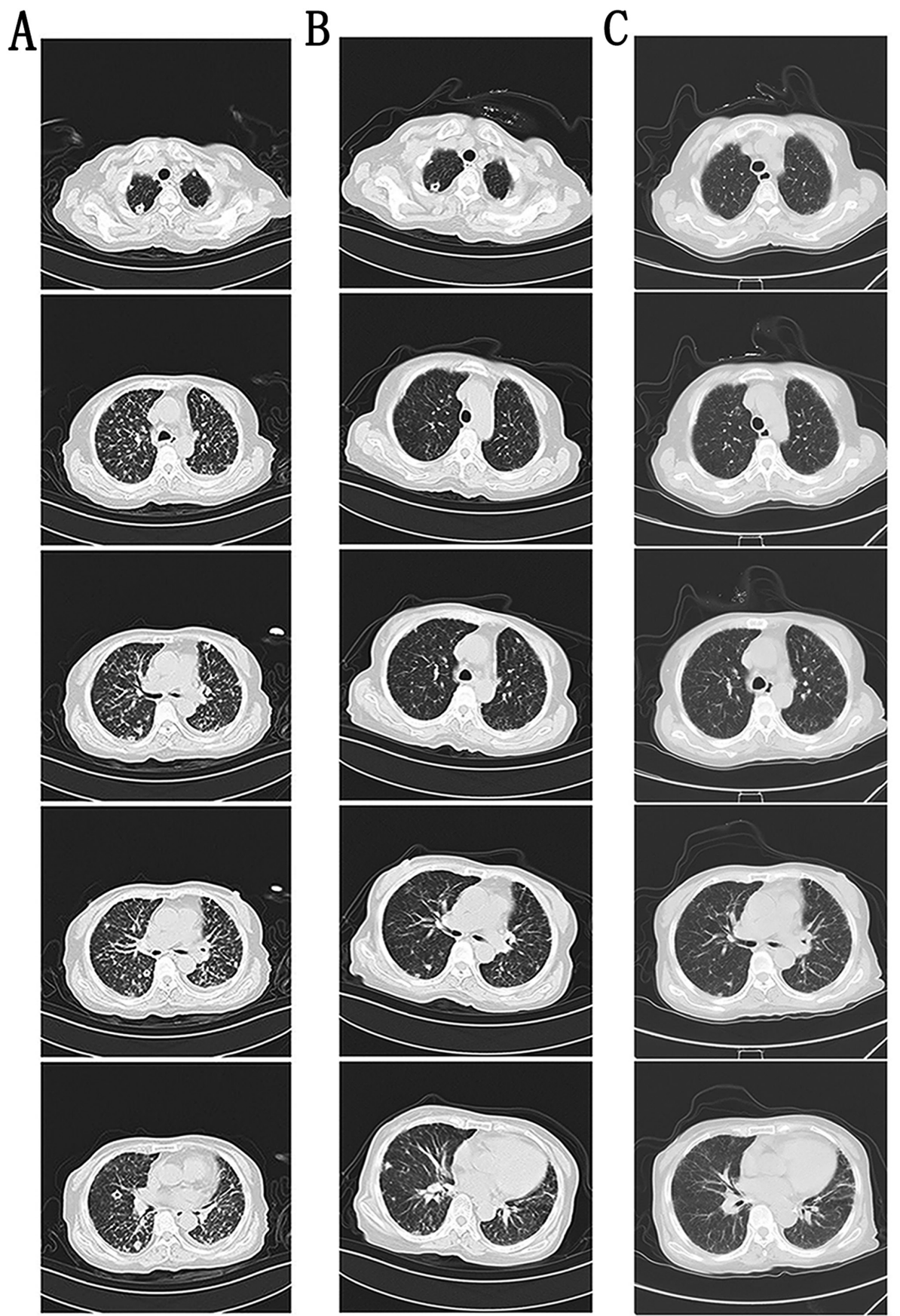

Figure 1. Changes shown by the chest CT scan before and after treatment. (A) Multiple irregular thick wall cavities, along with the mass, patch, cords and nodular shadows at the beginning are evident. (B) Cavities and lesions were significantly absorbed following treatment with linezolid, $0.6 \mathrm{~g}$, every $12 \mathrm{~h}$, at 10 days later. (C) The lesions had significantly shrunk in size in the chest CT scan before discharge. (A-C) The five images shown represent five different levels of the CT scan. CT, computed tomography.

not performed. Oral TMP-SMX treatment was terminated by the patient following discharge. The symptoms reappeared after 10 days, and the patient passed away two months later in a state of coma.

\section{Discussion}

Previous studies have reported that nocardiosis tends to develop in patients with underlying diseases, such as diabetes, malignancy, chronic obstructive pulmonary disease and immunosuppressive disease $(3,4)$. Nocardia can cause opportunistic infections in immunocompetent hosts via inhalation or direct inoculation through the skin (5-8). However, to date, at least to the best of our knowledge, there is no published report available regarding the risk factors of the long-term use of paracetamol associated with nocardiosis. The combination of paracetamol with codeine allows for marked pain relief and it is useful in controlling mild-to-moderate pain. The oral administration of codeine at the normal adult dose (30-60 mg every $4 \mathrm{~h}$ to a maximum of $240 \mathrm{mg}$ daily) causes no euphoria or respiratory depression and it is rarely addictive $(9,10)$.

Secondly, the imaging findings of pulmonary nocardiosis are usually characterized by multiple pulmonary infiltrations, pleomorphic alterations, nodular changes 


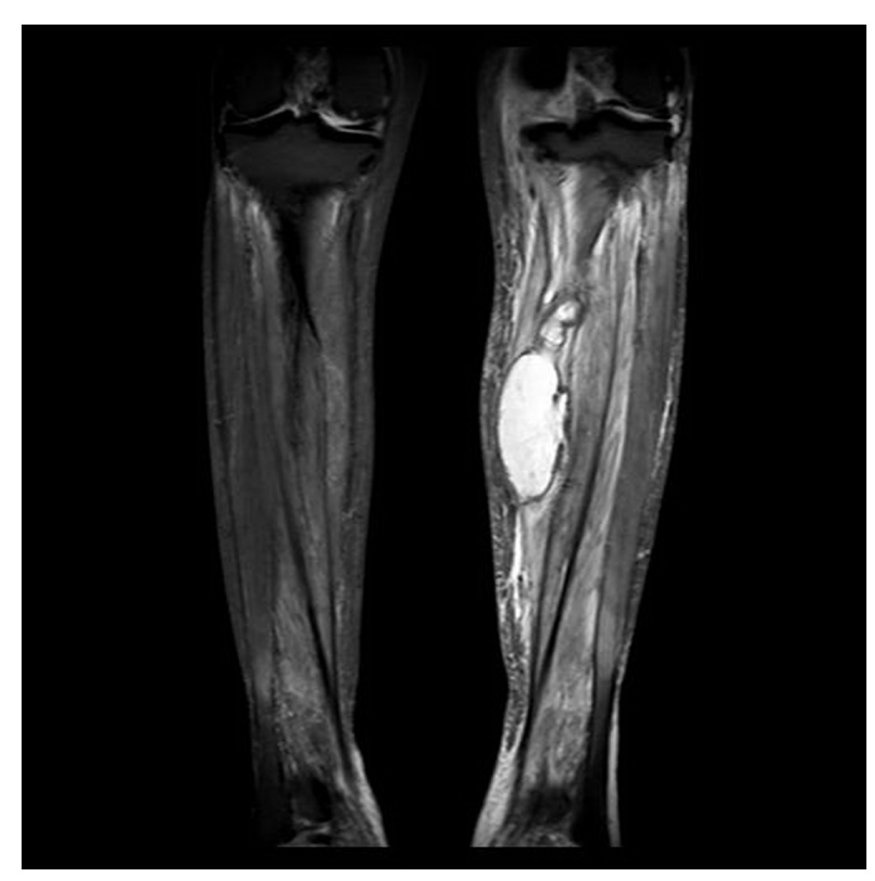

Figure 2. Magnetic resonance imaging (MRI) of the left leg. The image shows a high signal abscess intermuscular abscess in the left leg at the beginning when the patient visited Qinghai University Affiliated Hospital.

or cavity formation, and occasionally, by scattered small nodules, masses and interstitial alterations (11). However, this has not attracted sufficient research interest and has thus led to a delay in the early diagnosis of the disease. Only a few species (Nippostrongylus brasiliensis, Nocardia farcinica and Nocardia pseudobrasiliensis) can be reliably identified by traditional biochemical methods (12). However, although Nocardia was detected in the microbial culture of the patient described in the present study, a susceptibility test was not performed at Qinghai University Affiliated Hospital. It was suspected that the patient may have an underlying periodontal abscess that led to the secondary infection of Nocardia; however, symptoms such as headaches were relieved with the long-term administration of oral analgesics.

Finally, standard treatment may also affect the final outcomes. In line with previous findings (13), TMP-SMX is currently the main antimicrobial therapy for Nocardia. The most recent therapeutic alternative is linezolid, which exhibits $100 \%$ sensitivity to Nocardia species in vitro (14). The course of treatment should be at least three to six months for patients with normal immune function and needs to be extended to 12 months for patients with low immune function or central nervous system disease, according to the treatment guidelines (6). In the present study, linezolid and TMP-SMX treatment was terminated by the patient when the symptoms had improved, which may have led to the recurrence of the illness, thus causing her demise.

In conclusion, Nocardia infection is difficult to diagnose due to no typical clinical manifestations. It is imperative that the diagnosis be made early as possible. From the case presented herein, some experience was acquired, showing that the regular treatment of Nocardia may be beneficial for patients. The long-term use of paracetamol and codeine phosphate may be a risk factor for nocardiosis.

\section{Acknowledgements}

Not applicable.

\section{Funding}

No funding was received.

\section{Availability of data and materials}

The datasets used and/or analyzed during the current study are available from the corresponding author on reasonable request.

\section{Authors' contributions}

YL and HL provided the clinical data and collated the data for the present study. YS was responsible for the design and the writing of the study. YL and HL confirm the authenticity of all the raw data. All authors have read and confirmed the final manuscript.

\section{Ethics approval and consent to participate}

Not applicable.

\section{Patient consent for publication}

The patient information and images to be published was provided by a legally authorized representative. Written informed consent for the patient information to be published was obtained.

\section{Competing interests}

The authors declare that they have no competing interests.

\section{References}

1. Ambrosioni J, Lew D and Garbino J: Nocardiosis: Updated clinical review and experience at a tertiary center. Infection 38 : 89-97, 2010.

2. Lebeaux D, Bergeron E, Berthet J, Djadi-Prat J, Mouniée D, Boiron P, Lortholary $\mathrm{O}$ and Rodriguez-Nava V: Antibiotic susceptibility testing and species identification of Nocardia isolates: A retrospective analysis of data from a French expert laboratory, 2010-2015. Clin Microbiol Infect 25: 489-495, 2019.

3. Iida S, Kageyama A, Yazawa K, Uchiyama N, Toyohara T, Chohnabayashi N, Suzuki SI, Nomura F, Kroppenstedt RM and Mikami Y: Nocardia exalbida sp. nov., isolated from Japanese patients with nocardiosis. Int J Syst Evol Microbiol 56: 1193-1196, 2006.

4. Kato K, Noguchi S, Naito K, Ikushima I, Hanaka T, Yamasaki K, Kawanami T and Yatera K: Pulmonary nocardiosis caused by Nocardia exalbida in a patient with lung cancer and radiation pneumonitis: A case report and literature review. Intern Med 58: 1605-1611, 2019.

5. Bennett JE, Dolin R and Blaser MJ: Mandell, Douglas, and Bennett's Principles and Practice of Infectious Diseases: 2-Volume Set. Elsevier Health Sciences, 2014.

6. Wilson JW: Nocardiosis: Updates and clinical overview. Mayo Clin Proc 87: 403-407, 2012.

7. Chacón CF, Vicente R, Ramos F, Porta J, Lopez Maldonado A and Ansotegui E: Nocardia farcinica lung infection in a patient with cystic fibrosis and a lung transplant. Rev Esp Anestesiol Reanim 62: 161-164, 2015 (In English, Spanish).

8. Elenkov IJ: Glucocorticoids and the Th1/Th2 balance. Ann NY Acad Sci 1024: 138-146, 2004. 
9. Coulthard P, Bailey E and Patel N: Paracetamol (acetaminophen) for pain after oral surgery. Oral Surg 7: 81-86, 2014.

10. Patel N, Bailey E and Coulthard P: Opioids for pain after oral surgery. Oral Surg 7: 196-202, 2014.

11. Mehrian P, Esfandiari E, Karimi MA and Memari B: Computed tomography features of pulmonary nocardiosis in immunocompromised and immunocompetent patients. Pol J Radiol 80: 13-17, 2015.

12. Valdezate S, Garrido N, Carrasco G, Medina-Pascual MJ, Villalón P, Navarro AM and Saéz-Nieto JA: Epidemiology and susceptibility to antimicrobial agents of the main Nocardia species in Spain. J Antimicrob Chemother 72: 754-761, 2017.
13. Hashemi-Shahraki A, Heidarieh P, Bostanabad SZ, Hashemzadeh M, Feizabadi MM, Schraufnagel D and Mirsaeidi M: Genetic diversity and antimicrobial susceptibility of Nocardia species among patients with nocardiosis. Sci Rep 5: $17862,2015$.

14. Huang L, Chen X, Xu H, Sun L, Li C, Guo W, Xiang L, Luo G, Cui Y and Lu B: Clinical features, identification, antimicrobial resistance patterns of Nocardia species in China: 2009-2017. Diagn Microbiol Infect Dis 94: 165-172, 2019. Attribution 4.0 International (CC BY 4.0) License. 